\title{
Solar Variability and its Terrestrial Effects
}

\author{
By GEORGE L. WITHBROE AND \\ WOLFGANG KALKOFE $\mathbf{N}^{1,2}$
}

\author{
${ }^{1}$ Space Physics Division, NASA Headquarters, Washington, DC 20546, USA \\ ${ }^{2}$ Harvard-Smithsonian Center for Astrophysics, Cambridge, MA 02138, USA
}

The solar output in radiation and particles varies on an eleven-year time scale, affecting terrestrial temperatures and perhaps even climate. We examine some of the data that support this contention.

\section{Introduction}

The Sun varies on many time scales. They may be as short as seconds or less for flares; weeks, for phenomena linked to the solar rotation; decades, for phenomena associated with the activity cycle and the magnetic cycle; or centuries and longer, for variations on time scales of ice ages and warm interglacial periods.

While we may experience the effects of events of the shortest to intermediate time scales, we must rely on the historical and geological records to tell us about the longest. For the shortest time scales we observe the explosive phenomena directly in the outermost layers of the Sun, both from the ground in visible light and, much more dramatically, from space in very high energy radiation and relativistic particles; for the longest time scales, we can observe the behavior of stars that are solar twins in most respects yet differ from the Sun in the level of their luminosity variation and, thus, exhibit behavior that the Sun may have shown in the past or may show in the future.

In this introductory paper we will merely touch upon some of the topics associated with these time scales. In Section 2 we discuss the variation in the solar output in relation to the eleven-year solar cycle, in Section 3 we consider the effects of solar variability on terrestrial surface temperatures and climate, and in Section 4 we compare the Sun to other stars; in the final section we draw some conclusions.

\section{Solar variability}

The most conspicuous phenomenon of solar variability is the appearance and disappearance of dark spots on the face of the Sun, which occurs at the rhythm of ten to twelve years (Figure 1) and an average (during the last three centuries) of eleven years. Sunspots mark the waxing and waning of solar activity. At the same time, the polarity of the solar magnetic field reverses, so the magnetic cycle is twice as long as the activity cycle. As is evident from Figure 1, which shows the sunspot number (a measure of activity that depends on the number of individual spots and the number of groups of spots), the length of the cycle is not sharply defined, nor is the level of activity. Another indicator of the solar cycle is the radio flux at a wavelength of $10.7 \mathrm{~cm}$. Its smoothed variation matches very well the behavior of the sunspot number, and is another convenient measure of solar activity. There is a hint that a rapid rise of activity leads to a short cycle and a high maximum and, thus, to a relation between cycle length and activity level at solar maximum (cf. also Figure 5).

Near solar maximum, the level of emission from the hot outermost layers of the Sun rises. This can be seen in Figure 2, which shows the variation in the flux at several wavelengths late during the rising phase towards the maximum of cycle 21 . The dominant 


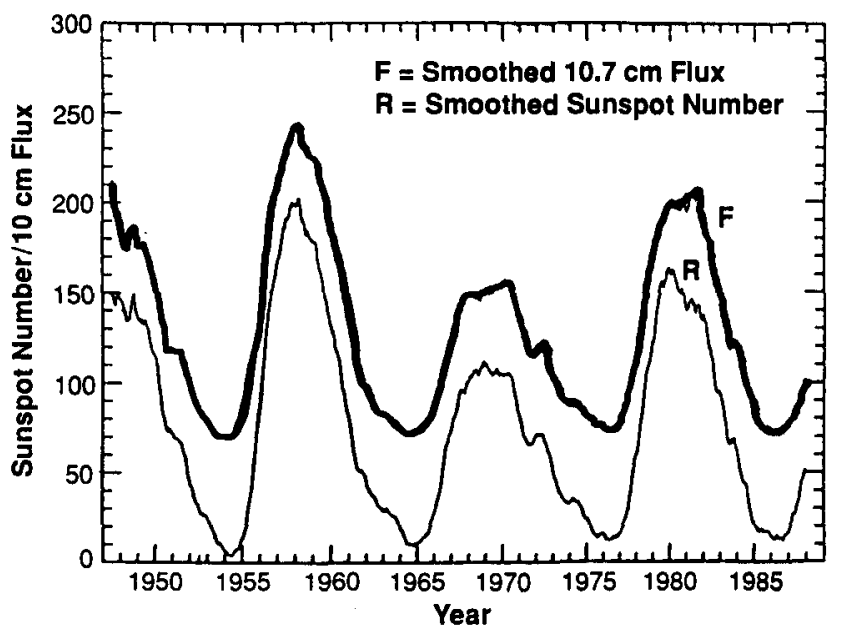

Figure 1. Smoothed (13-month running mean) sunspot number, $R$, and solar $10.7 \mathrm{~cm}$ radio flux for solar cycles 19,20 , and 21 (Withbroe 1990).

variation is that of the 27-day solar rotation, but a distinct increase in the emission maxima is also evident. Most of the emission occurs in the ultraviolet part of the spectrum, as shown in the right-hand panels of the figure. At Lyman- $\alpha$, for example, the increase in emission at solar maximum over that at minimum may be as much as a factor of two, while at $\mathrm{X}$-ray wavelengths it can be more than two orders of magnitude. It is interesting to note that, for this rotational modulation of the emission, the maxima of the short wavelength radiation from the hot corona coincide approximately with the minima in the total irradiance (cf. Lean 1991, Figure 14). Thus, sunspots cause a short-term deficit in total irradiance when they are directly facing the Earth. Even though the extra emission at short wavelengths also peaks at that time, it is insufficient to compensate this sunspot deficit. Nevertheless, despite this short-term shortfall in solar output, total irradiance averaged over a solar rotation is maximal at sunspot maximum (cf. Figure 7). The source of the excess radiation is unknown.

The frequency of optical flares follows the sunspot number (cf. Figure 3), increasing by more than an order of magnitude at sunspot maximum over that at minimum. Flares are explosive events occurring in the very hot, tenuous outer layers of the solar atmosphere. They generate copious amounts of high-energy photons and particles, including relativistic elementary particles with energies beyond $10 \mathrm{MeV}$. Large flares, and especially large $\mathrm{X}$-class flares which emit prodigious quantities of radiation, tend to occur in the declining phase of the solar cycle beyond maximum. The frequency of proton events (cf. Figure 3) follows this behavior, as do geomagnetic storms, which are responses of the Earth's magnetosphere to coronal mass ejections and high-speed solar wind streams from low-latitude coronal holes. Geomagnetic storms can cause catastrophic failures of electric power grids. In the upper atmosphere of the Earth, where high-energy radiation and particles from flares are absorbed, they modify the photochemistry as well as the temperature and density structure of the atmosphere. Large flares affect satellites in two 

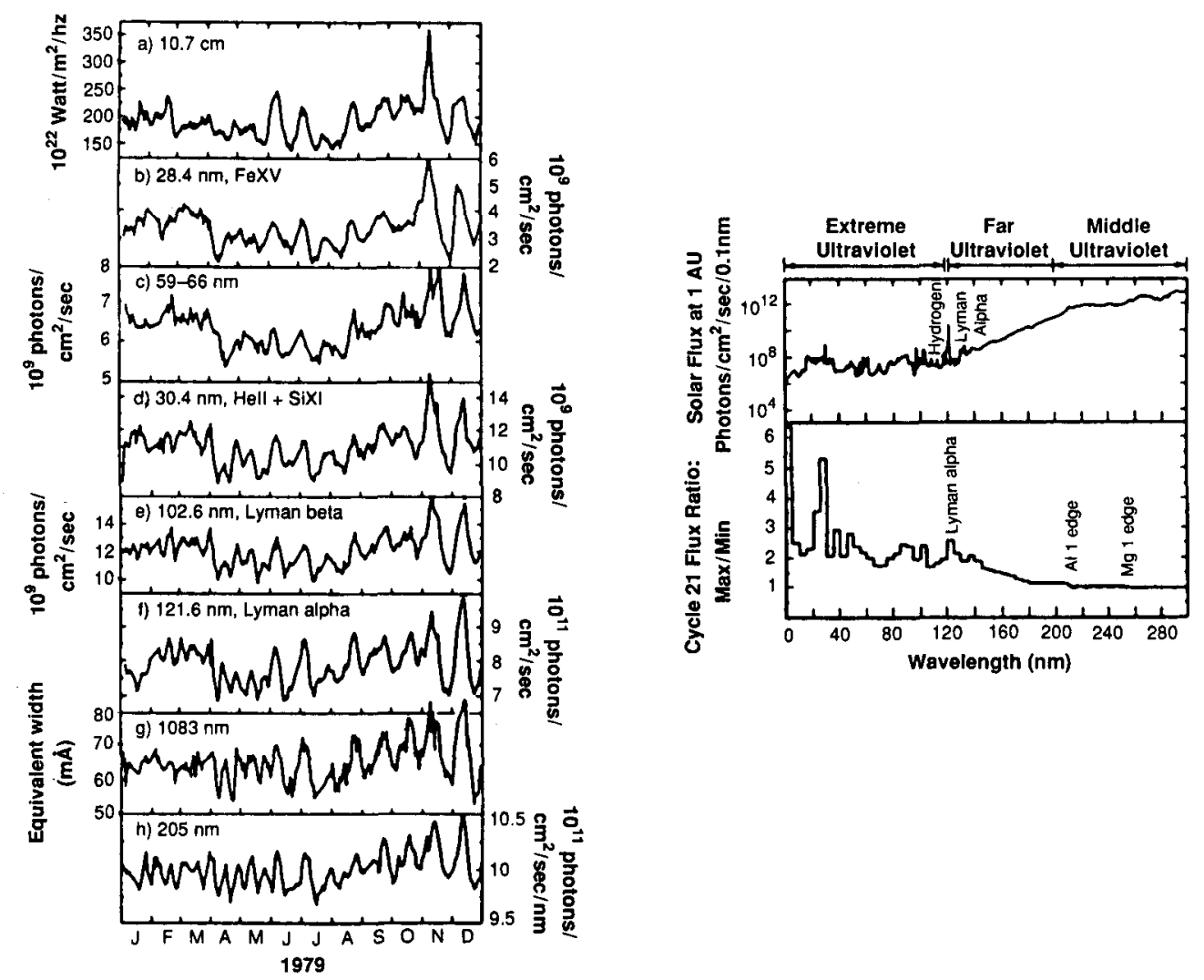

Figure 2. Left: Solar variability in 1979 at $10.7 \mathrm{~cm}$ in the radio band, $10,830 \AA$ in the infrared, and at several UV and EUV wavelengths. Right: The solar UV spectrum, and an estimate of the spectral variability shortward of $2900 \AA$ during cycle 21 (Lean 1987).

ways: by exposing them to intense, penetrating radiation, which could be dangerous to astronauts and biological systems, and by limiting the life times of satellites in low earth orbit through atmospheric heating and increased drag.

\section{Terrestrial surface temperatures and climate}

As is evident from Figure 1, the solar cycle is irregular, both in length and in intensity. We may ask what the extent of the variability of the Sun has been in the past, and what it might be for a star like the Sun in the future. Considering the first question, we see in Figure 4 the sunspot number from 1600 to the present time (Lean 1991). The solid line represents monthly means and the dashed line and crosses, yearly means. The data clearly show the alternation between maxima and minima in the sunspot number. While the length of the activity cycle is quite well defined by the data from the last three centuries as close to 11 years, the amplitude of the sunspot number varied by as much as a factor of five. Yet sunspots never failed to appear at the expected time of solar maximum. It is different for the preceding half century, the so-called Maunder Minimum, when sunspots were either few in number or completely absent. The cycle length is therefore undefined in the data plotted. However, Nesme-Ribes reports at this 

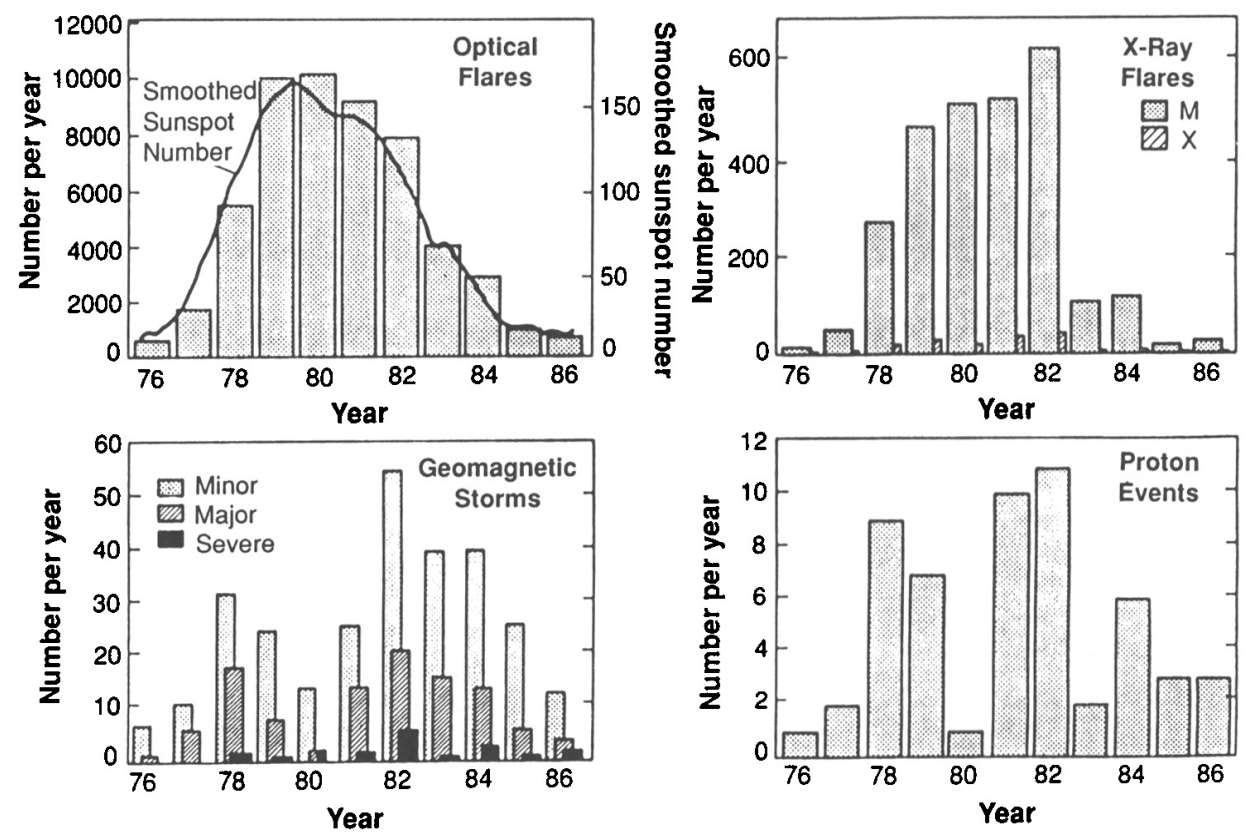

Figure 3. Frequency of flares, geomagnetic storms and proton events during maximum of cycle 21 .

conference that the length of the cycle during the Maunder Minimum was 9 or 10 years. This combination of a short cycle length and very low activity is surprising in view of the usual result that a short cycle length implies an active maximum.

According to Eddy (1976), "The 11 year sunspot cycle may not have been operative before about 1700 . Instead, solar activity appears to have been characterized by extended periods of either enhanced activity or inactivity. Thus during the 290 years when the Sun has been observed most intensely its behavior may have been unusually regular and benign." The quality of this conclusion parallels the finding (Anklin et al. 1993) from the Greenland Ice Core Project (GRIP) that abrupt and large climatic oscillations can occur during both glacial and interglacial periods, and that the past 8000 years have been strangely stable. Even though the periods discussed in the two papers are different and a definite connection between solar activity and climate has not been definitely established, circumstantial evidence suggests that the similarity of conclusions is not accidental. It is interesting to note here that approximately one third of the solar-like stars appear to be in a state analogous to the Maunder Minimum (cf Baliunas \& Jastrow 1990), which matches the fraction of time the Sun has been in a state of minimal activity with very low sunspot numbers during the last millenium (cf Figure 6). Thus, both the past behavior of the Sun and the present behavior of solar-like stars suggest that the stability in solar behavior we have witnessed during the last several centuries is unusual.

A relation between solar activity and climate is suggested by the data covering a century on sunspot number, cycle length and terrestrial surface temperatures displayed in Figure 5. There is an obvious correspondence between cycle length and land temperature in the northern hemisphere and, similarly, between sunspot number and global sea temperature. Apart from the impression that the (inverse) cycle length peaks earlier than does the sunspot number, one would conclude that a short cycle length corresponds to a strong maximum in sunspot number. It is interesting to note again the apparent contra- 


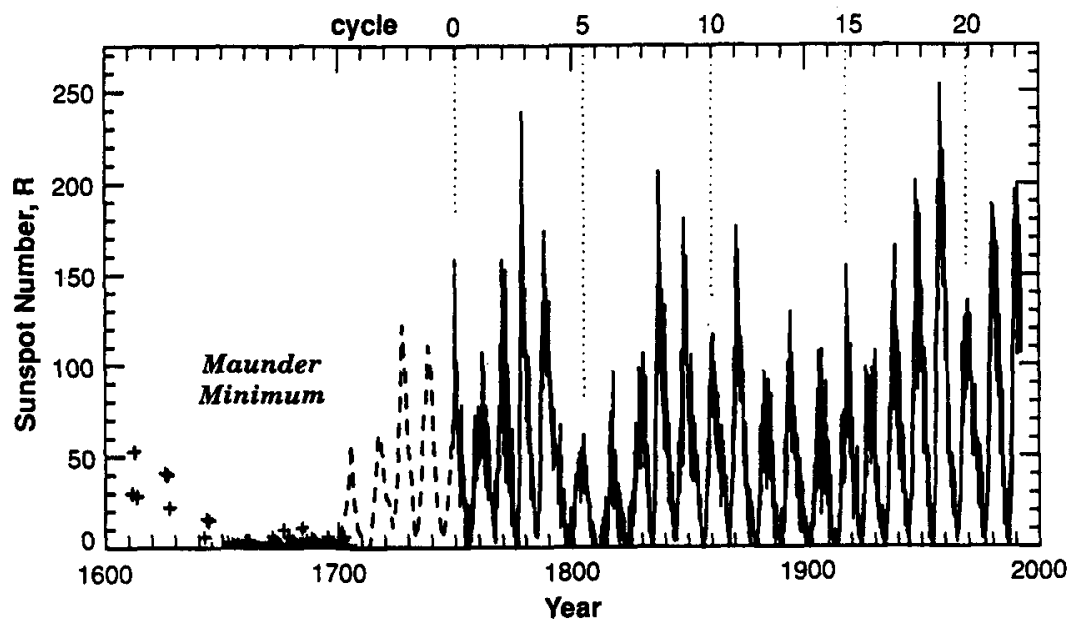

FIGURE 4. Sunspot number from 1600 to the present time.
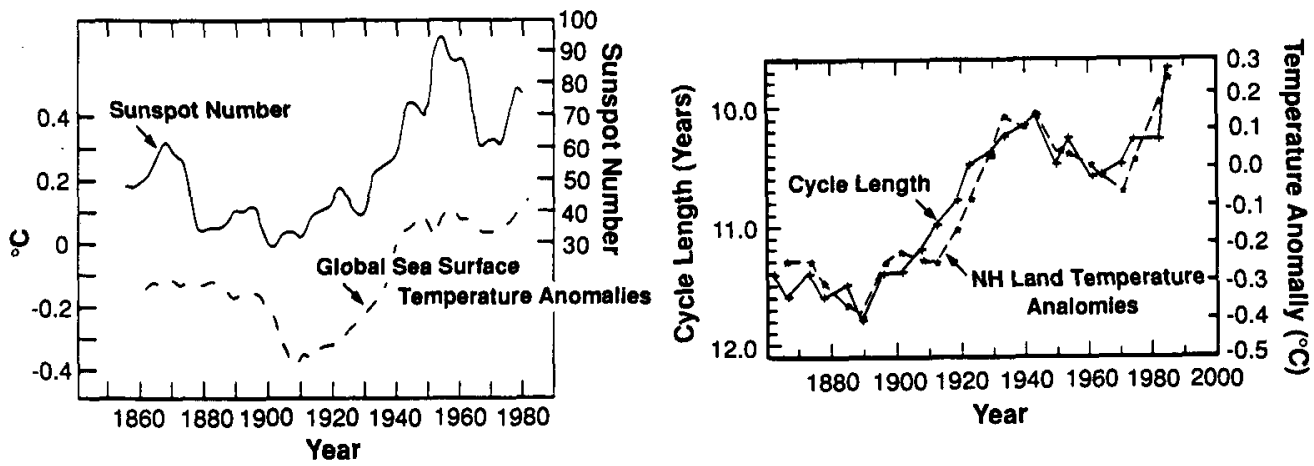

FIGURE 5. Comparison (left) of 11 year running mean of the sunspot number with global average sea surface temperature anomalies (Reid 1991), and (right) length of solar cycle, from smoothed sunspot extrema, with northern hemisphere temperature anomalies (Friis-Christensen \& Lassen 1991).

diction that the cycle length appears to have been unusually short during the Maunder Minimum. The data displayed in Figure 5 would have suggested land and sea temperatures higher than usual at that time. But it is known from the historical record as well as from Dutch landscape paintings that temperatures were uncommonly low, giving the period the name of Little Ice Age. Other observations showing a close relation between solar activity and climate are, for example, the amount of rainfall in several nothern latitude bands over the last century (cf. Noyes $1982 \mathrm{Ch} .10$ ): The rainfall was correlated with the sunspot number in the latitude band from $70^{\circ}$ to $80^{\circ}$, anticorrelated from $60^{\circ}$ to $70^{\circ}$, and changed correlation in the band from $50^{\circ}$ to $60^{\circ}$.

Figure 6 makes again the case for a connection between solar activity and climate in northern Europe. Several measures of solar activity over the last millenium are indicated: ${ }^{14} \mathrm{C}$ variation (lower line and open circles), a qualitative measure of the severity of winter (upper line, shifted right to allow for the carbon isotope to be assimilated into tree rings), the sunspot number since 1700 , and naked-eye sunspot observations (dark circles); the 


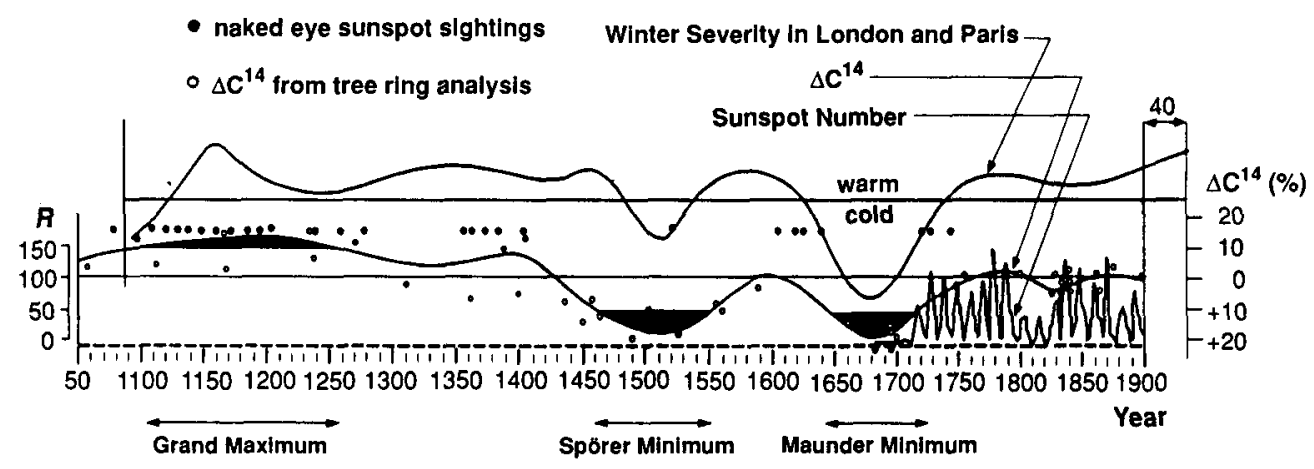

FIGURE 6. Relation between the severity of winter and solar activity (from Eddy 1976, 1977).

Maunder and Spörer Minima and the Medieval Maximum are indicated by shading. Qualitatively, the general correspondence between climate, as perceived in London and Paris, and solar activity, as indicated by organic carbon deposits and sunspot number, is evident.

\section{Solar and stellar variability}

A means by which the Sun can influence climate is the variation of the total irradiance. The question is whether the measured irradiance variation is sufficiently large. As Figure 5 showed, solar activity correlates with terrestrial surface temperature. However, it is generally believed that the current amplitude of the variation of total irradiance of $0.1 \%$ can have only a negligible effect on climate. Figure 7 displays total solar irradiance over approximately one cycle, as well as sunspot number. It is noteworthy that although both ERB and ACRIM agree on the variation of $0.1 \%$, they differ on the level of the irradiance by $0.3 \%$. This worrisome fact would not have been discovered without overlapping coverage, which points to the necessity of having a second instrument commence measurement before the first one ends operation.

The expectation that an irradiance change of $0.1 \%$ cannot affect climate appears to be inconsistent with the implications of the obvious correspondence between the low level of solar activity during the Maunder Minimum and the low temperatures experienced in many parts of the world. However, it is thought that solar irradiance during the Maunder Minimum was depressed not by the usual $0.1 \%$, but by $0.3 \%$, and during an extended period of time (see also papers in this volume by Lean et al. and Nesme-Ribes et al.). This raises the question of the range of luminosity variation that can be expected from a star like the Sun.

Figure 8 gives the histogram of magnetic activity for solar-analog stars as measured by calcium emission. An HK index greater than 0.17 corresponds to the active Sun, and smaller than 0.17 to the quiet Sun. The Sun thus appears to show average behavior as far as chromospheric emission is concerned. The same conclusion is reached by Lockwood (1994) who notes that in the Mount Wilson sample of stars, the Sun fits into the middle of the distribution of calcium indices. However, he also reports on the luminosity varation of solar-type stars observed at Lowell Observatory. In this sample, the Sun joins a minority 


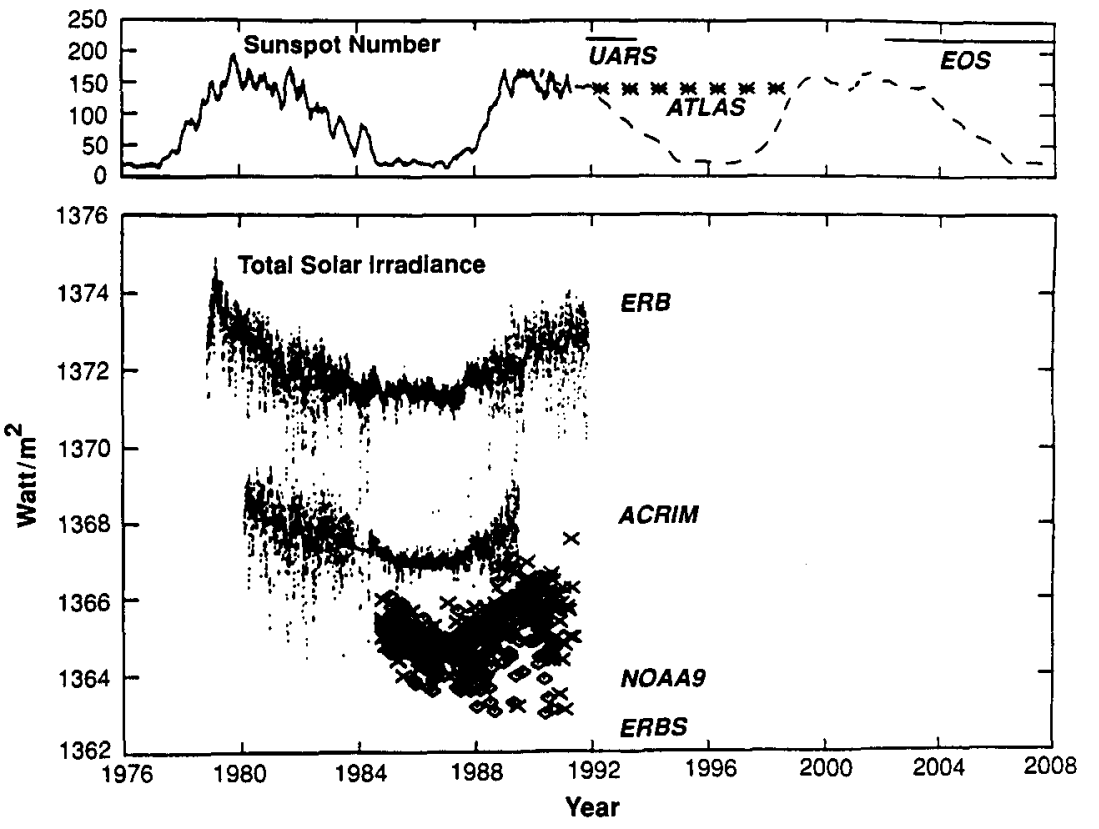

FIGURE 7. Solar activity variation measured by sunspot number (top), and by the ERB radiometer on the Nimbus-7 satellite and ACRIM on SMM, and by the ERBE program on NOAA9 and ERBS (Lean, private communication).

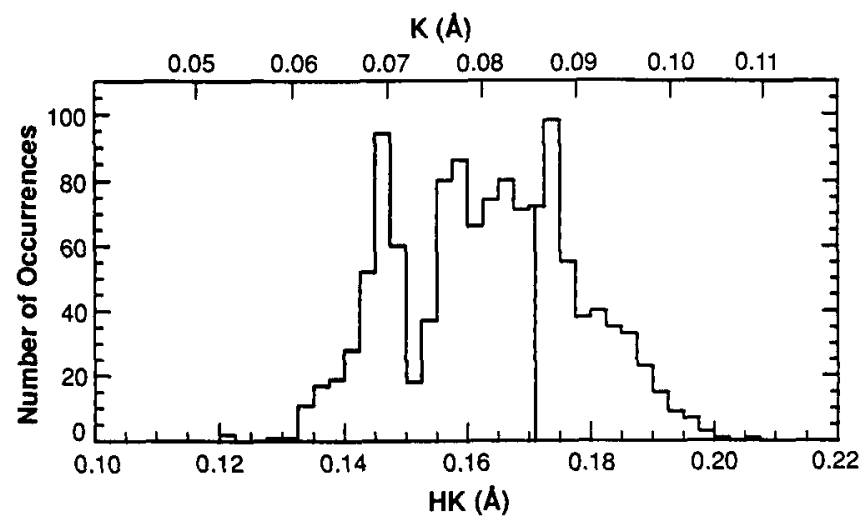

Figure 8. Frequency distribution of solar-like stars as determined from emission in the $\mathrm{H}$ and $\mathrm{K}$ lines of $\mathrm{Ca}$ II, and in the $\mathrm{K}$ line alone (Baliunas \& Jastrow 1990).

of stars with a low luminosity variation of $0.1 \%$. Most of the stars vary by $0.3 \%$, and some by as much as $1 \%$. These results suggest that the larger luminosity variation of $0.3 \%$ inferred for the Sun during the Maunder Minimum would be typical behavior for stars that are like the Sun in mass, composition and rotation period and, therefore, in the activity of their magnetic dynamo. Thus, a substantially larger solar variation than the current $0.1 \%$ is consistent with the stellar observations.

It may be worth noting that the study of solar-analog stars may be essential in pointing 
up differences in various features, such as chromospheric and coronal emission, luminosity, and phase relations between maximal brightness and maximal color index (cf Gray 1994). Such differences may help elucidate the physical mechanism underlying solar and stellar variability.

\section{Conclusions}

Solar activity and global temperatures appear to be highly correlated. However, according to studies with general circulation models the current level of irradiance variation is too low to change climate. A higher level of solar variability appears possible and, as the study of solar-type stars indicates, even likely. The societal consequences of a change in climate are expected to be enormous. In order to assess the relative importance of solar variability and of anthropogenic causes it is imperative that we investigate and understand the influence of the Sun alone. This requires a long-term program of monitoring total and spectral irradiance and an effort to study the physical mechanism of solar and stellar luminosity variation. The stars are important in this context as well since different phenomena observed in stars may help us identify the unknown source of excess radiation in the Sun at activity maximum and point to the underlying cause of solar as well as stellar variability.

Acknowledgments. We are grateful to Drs. Judith Lean and William Wagner for helpful discussions and critical reading of the manuscript.

\section{REFERENCES}

ANKLIN, M. ET AL. 1993 Climate instability during the last interglacial period recorded in the GRIP ice core. Nature 364, 203-207.

Baliunas, S. \& Jastrow, R. 1990 Evidence for long-term brightness changes of solar-type stars. Nature 348, 520-523.

Eddy, J. A. 1976 The Maunder Minimum. Science 192, 1189-1202.

EDDY, J. A. 1977 Historical evidence for the existence of the solar cycle. In The Solar Output and its Variation (ed. O.R. White). pp. 51-71. Assoc. Univ. Press, Boulder, CO.

Friss-Christensen, E. \& LASSEN, K. 1991 Length of the solar cycle: an indicator of solar activity closely associated with climate. Science 254, 698-700.

GraY, D. 1993 Variations during stellar cycles. Contributed paper presented at IAU Colloquium No. 143 "The Sun as a Variable Star: Solar and Stellar Irradiance Variations", June 20-25, 1993, Boulder, Colorado.

LEAN, J. 1987 Solar ultraviolet irradiance variations: A review. J. Geophys. Res. 92, 839-868.

LEAN, J. 1991 Variations in the Sun's radiative output. Rev. Geophys. 29, 505-535.

Lean, J., Skumanich, A., White, O.R. \& Rind, D. 1994 Estimating solar forcing of climate change during the Maunder Minimum. In The Sun as a Variable Star: Solar and Stellar Irradiance Variations (ed. J.M. Pap, C. Fröhlich, H.S. Hudson \& S.K. Solanki). Cambridge University Press, in press.

Lockwood, G.W. 1994 Irradiance variations of stars. In The Sun as a Variable Star: Solar and Stellar Irradiance Variations (ed. J.M. Pap, C. Fröhlich, H.S. Hudson \& S.K. Solanki. Cambridge University Press, in press.

Nesme-Ribes, E., SokolofF, D. \& SADourny, R. 1994 Solar rotation, irradiance changes and climate. In The Sun as a Variable Star: Solar and Stellar Irradiance Variations (ed. J.M. Pap, C. Fröhlich, H.S. Hudson \& S.K. Solanki). Cambridge University Press, in press.

Noyes, R. W. 1982 The Sun, Our Star, Harvard Univ. Press, Cambridge, MA. 
G. L. Withbroe \& W. Kalkofen: Solar Variability and its Terrestrial Effects

REID, G. C. 1991 Solar total irradiance variation and the global sea surface temperature record. J. Geophys. Res. 96, 2835-2844.

Withbroe, G. L. 1990 Adv. in Astronaut. Sc., Vol. 71. 